\title{
Approximate solutions for non-linear iterative fractional differential equations
}

\begin{abstract}
This paper establishes approximate solution for non-linear iterative fractional differential equations: $\operatorname{dyv}(\mathrm{s}) / \mathrm{d} s \mathrm{~s}=\aleph(\mathrm{s}, \mathrm{v}, \mathrm{v}(\mathrm{v}))$, where $\supset \in(0,1], \mathrm{s} \in \mathrm{I}:=[0,1]$. Our method is based on some convergence tools for analytic solution in a connected region. We show that the suggested solution is unique and convergent by some well known geometric functions.
\end{abstract}

Keyword: Non-linear iterative fractional differential equations; Solutions 